\title{
Colletotrichum species associated with mango in southern China
}

\begin{abstract}
Qili $\mathrm{Li}^{1}$, Junyan $\mathrm{Bu}^{2}$, Juan Shu ${ }^{2}$, Zhihe $\mathrm{Yu}^{2 *}$, Lihua Tang ${ }^{1}$, Suiping Huang ${ }^{1}$, Tangxun Guo ${ }^{1}$, Jianyou $\mathrm{Mo}^{1^{*}}$, Shuming $\mathrm{Luo}^{3}$, Ghulam Sarwar Solangi $\mathbb{B}^{4}$ \& Tom Hsiang $\mathbb{B}^{5}$

Mango (Mangifera indica L.) is an economically significant fruit crop in provinces of southern China including Hainan, Yunnan, Sichuan, Guizhou, Guangdong and Fujian. The objective of this study was to examine the diversity of Colletotrichum species infecting mango cultivars in major growing areas in China, using morphological and molecular techniques together with pathogenicity tests on detached leaves and fruits. Over 200 Colletotrichum isolates were obtained across all mango orchards investigated, and 128 of them were selected for sequencing and analyses of actin (ACT), chitin synthase (CHS-1), glyceraldehyde-3-phosphate dehydrogenase (GAPDH), the internal transcribed spacer (ITS) region, $\beta$-tubulin (TUB2) genomic regions. Our results showed that the most common fungal isolates associated with mango in southern China involved 13 species: Colletotrichum asianum, C. cliviicola, C. cordylinicola, C. endophytica, C. fructicola, C. gigasporum, C. gloeosporioides, C. karstii, C. liaoningense, C. musae, $C$. scovillei, $C$. siamense and $C$. tropicale. The dominant species were $C$. asianum and $C$. siamense each accounting for $30 \%$, and C. fructicola for $25 \%$. Only C. asianum, C. fructicola, C. scovillei and $C$. siamense have previously been reported on mango, while the other nine Colletotrichum species listed above were first reports associated with mango in China. From this study, five Colletotrichum species, namely C. cordylinicola, C. endophytica, C. gigasporum, C. liaoningense and C. musae were the first report on mango worldwide. Pathogenicity tests revealed that all 13 species caused symptoms on artificially wounded mango fruit and leaves (cv. Tainong). There was no obvious relationship between aggressiveness and the geographic origin of the isolates. These findings will help in mango disease management and future disease resistance breeding.
\end{abstract}

Mango (Mangifera indica L.) is one of the most economically important fruit crops worldwide, and after India, China is the second largest mango producer ${ }^{1}$. The planted area of mango in China was approximately 173,000 ha and the total production was $1,437,700$ tons on the mainland in $2014^{2}$. Major mango producing areas in China include Guangdong, Guangxi, Guizhou, Fujian, Hainan, Yunnan and Sichuan provinces. Mango anthracnose caused by several Colletotrichum species, has been considered the most important disease on mango in China ${ }^{3}$. Annually mango anthracnose could be liable for about $30-60 \%$ loss of production, and the damage could be $100 \%$ if optimum conditions exist for the pathogen ${ }^{4}$.

Traditionally, Colletotrichum species are identified based on morphological characters including conidial and appressorial size and shape, presence of acervuli, setae, or sclerotia, sexual structures, and cultural characters such as growth rate and colony color ${ }^{5}$. Distinguishing Colletotrichum species within the genus morphologically has been difficult due to their phenotypic similarity, and the fact that variable environmental factors could also affect expression of morphological traits. Frequently, all available morphological characteristics are combined and used for systematic species discrimination ${ }^{6}$.

Studies on Colletotrichum have classified the genus into nine major clades plus isolated species or small clusters, with clades probably representing species complexes ${ }^{7}$. The internal transcribed spacer region (ITS) of ribosomal DNA has been frequently used to separate Colletotrichum species ${ }^{8}$, but sometimes the ITS sequence information alone was not enough for clarifying species boundaries within the genus ${ }^{7}$. Thus, other genes including ACT, CHS-1, GAPDH and TUB2 have been used to resolve relationships among many fungi, including Colletotrichum species ${ }^{9}$. A multiple evidence approach, using combined molecular sequencing information and

${ }^{1}$ Institute of Plant Protection, Guangxi Academy of Agricultural Sciences and Guangxi Key Laboratory of Biology for Crop Diseases and Insect Pests, Nanning, Guangxi, 530007, China. ${ }^{2}$ College of Life Sciences, Yangtze University, Jingzhou, Hubei, 434025, China. ${ }^{3}$ Plant Breeding Institute, The University of Sydney, Cobbitty, NSW, 2570, Australia. ${ }^{4}$ Department of Entomology, Sindh Agriculture University Sub-campus, Umerkot, 69100, Pakistan. ${ }^{5}$ Environmental Sciences, University of Guelph, Guelph, Ontario, Canada. *email: zhiheyu@hotmail.com; mojianyou@gxaas.net 
morphological data is now recommended as an alternative method for accurate species identification in the genus Colletotrichum $^{6,7,9}$.

Colletotrichum species complexes such as C. acutatum, C. amagnum, C. aorchidearum, C. boninense, C. dracaenophilum and C. gloeosporioides have been separated into many different species using morphological and molecular techniques ${ }^{9-13}$. However, in China the identification of Colletotrichum species on mango has relied mainly on conidial morphology and ITS sequences ${ }^{14}$, and the combined data were still limited and insufficient to distinguish closely related taxa in the six species complexes mentioned above. Previous research regarding Colletotrichum species on mango were mostly samples from small and fragmented provincial areas, with poor representation of the actual species diversity in the vast mango growing regions in China.

In our previous study, three Colletotrichum species causing mango anthracnose in Guangxi province in China (C. asianum, C. fructicola and C. siamense) were reported ${ }^{15}$. However, the growing season and the harvesting time of mango in seven provinces growing mangoes vary in environment, climatic conditions and varieties grown, which may lead to variations in composition and distribution of causal agents of mango anthracnose.

Genetic diversity is often related to evolutionary potential, and an ability to adapt to variable environmental conditions ${ }^{16}$. Based on our current understanding of the gene-for-gene hypothesis, resistance genes lose their effectiveness when faced with complex and evolving pathogen populations. An understanding of the population structure and species distribution of pathogens can provide insights into optimal breeding strategies for durable resistance to anthracnose in mangoes and improved cultural controls.

The objectives of this study were as follows: (1) to identify Colletotrichum species associated with mango anthracnose symptoms in six provinces of China using cultural characteristics such as conidial and appressorial morphology as well as multi locus sequencing data for phylogenetic analyses; (2) to test the aggressiveness of isolates from different geographic locations in the major mango growing areas in mainland China; and (3) to examine the variation of isolates within and between regions.

\section{Results}

Fungal isolations. In 2016 and 2017, all the leaves, fruits and branches of mango with anthracnose symptoms collected from the six mango-growing provinces in China were used for fungal isolation. Based on morphology observation and ITS sequences, a total of 205 Colletotrichum isolates were obtained from all mango growing regions investigated, and 128 representative isolates were selected for further study (one isolate from each orchard, and more than one for some big orchards; 34 from Yunnan, 23 from Hainan, 18 from Guizhou, 13 from Sichuan, 16 from Guangdong and 24 from Fujian province). The selected isolates are listed in Supplementary Table S1. Isolated species in other genera include Alternaria, Phomopsis, Botryosphaeria, and Pestalotia and were retained for further study.

Phylogenetic analyses. These isolates from different areas were used for sequencing and analyses of ACT, CHS-1, GAPDH and TUB2 loci. All sequences were first compared with the NR database from NCBI-GenBank using BLASTn, and then submitted to GenBank (see Supplementary Table S1). For phylogenetic analysis, sequences of extype or ex-epitype strains of Colletotrichum species (Supplementary Table S2) were selected. According to the ILD test, partitions of the data into ACT, CHS-1, GAPDH, ITS and TUB2 were homogeneous, and the data sets were combined for Maximum likelihood $(\mathrm{ML})$ and Bayesian analyses. For the Bayesian analyses, a JC model was selected for ACT, a GTR + I + G model for GAPDH, ITS and CHS-1, a HKY + G model for TUB2 and incorporated in the analysis.

Concatenated sequences from the five loci (ACT, TUB2, CHS-1, GAPDH and ITS) were generated for each of the 128 Colletotrichum isolates, plus ex-type or type strains downloaded from GenBank to generate a phylogenetic tree (Fig. 1). As the topology of the Bayesian analysis of the combined data set was nearly identical to that of the ML consensus tree, only the Bayesian tree is shown with bootstrap (from ML analysis) on the left and posterior probability (from Bayesian analysis) on the right of corresponding nodes (Fig. 1).

The results showed that 38 isolates were clustered with four C. asianum strains (ICMP 18605, ICMP 18603, IMI 313839 and ICMP 18580) showing 100\% posterior probability (PP) and bootstrap (BS); 38 isolates were aligned with five C. siamense strains (with $100 \%$ posterior probability and $95 \%$ bootstrap); and 32 isolates were placed with two C. fructicola strains (ICMP 18727 and ICMP 18613) and one C. ignotum strain (CBS125397) supported with $100 \%$ posterior probability and $90 \%$ bootstrap. Five isolates clustered with strains of $C$. karstii CBS118401, CBS 128524 and CBS 128500, with 100\% support; four isolates clustered with the type strain of C. endophytica CAUG28 with 100\% support; three isolates clustered with C. scovillei strains CAUA1, CBS 126529 and CBS 120708 with 100\% support, isolates YN8-2 and YN31-4 with type strain C. cliviicola CBS 125375 with $100 \%$ support; isolate HN42-2 with type strain of C. gigasporum CBS 133266, MUCL 44947 with 100\% support and isolate HN32-1 with type strain C. tropicale CBS 124949 with 100\% probability and 99\% bootstrap support. Isolate GZ14-G-1 clustered with type strain C. gloeosporioides IMI 356878 with $100 \%$ probability and $95 \%$ bootstrap support, isolate YN33-1 with type strain C. liaoningense CAUOS2 with 100\% support, HN23-5 with type strain of C. cordylinicola ICMP 18579 (100\% for PP and 88\% for BS) and GZ23-3 with type strain of C. musae ICMP 19119 (100\%).

Morphological observations. The colonies of the Colletotrichum isolates were white initially, later turning pale gray, with sparse aerial mycelium, hairy, sometimes producing abundant orange (\#FF8040) pionnotes (Fig. 2). There was high variation in mycelial growth rate among the 128 Colletotrichum isolates (Supplementary Table S3) with averages for C. asianum at $0.38-1.59 \mathrm{~cm} /$ day, C. siamense, $0.52-1.51 \mathrm{~cm} /$ day and C. fructicola, $0.60-1.19 \mathrm{~cm} /$ day.

Conidia were all hyaline, aseptate and long elliptic to cylindrical. The average conidial sizes (Supplementary Table S3) varied among species. For isolates grown on PDA, the length of conidia for each isolate varied from 6.99 


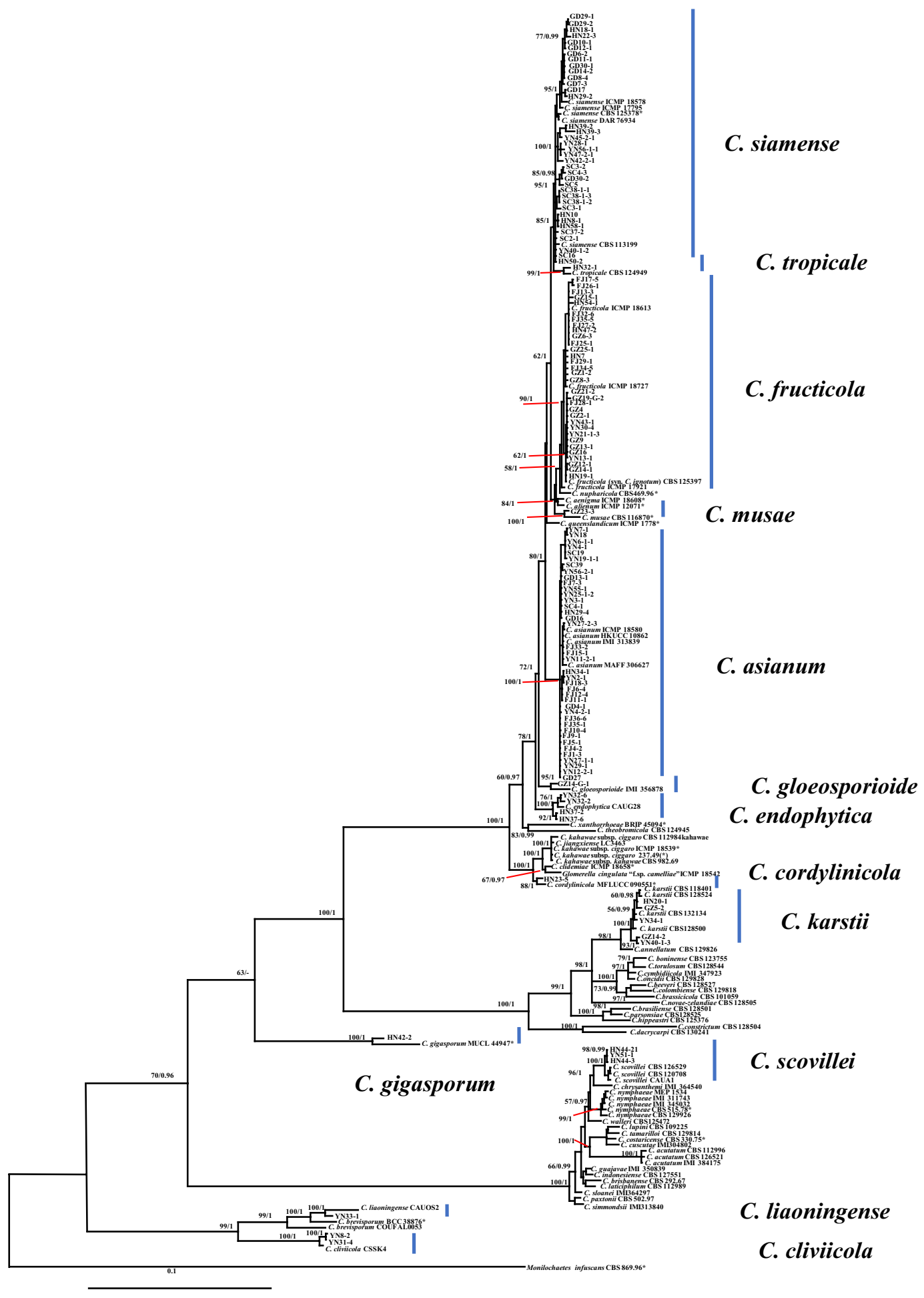

Figure 1. The phylogenetic tree was constructed using sequences of 128 Colletotrichum isolates, extype or exepitype isolates in the GenBank based on combined ITS, ACT, GAPDH, TUB2, and CHS-1 genomic data. The numbers on the left and right of each node are posterior probabilities estimated using the software MrBayes v. 3.2.6 and bootstrap values from RAxMLGUI v. 1.3.1, respectively.

$\mu \mathrm{m}$ to $29.22 \mu \mathrm{m}$, and the width of conidia varied from $2.99 \mu \mathrm{m}$ to $9.85 \mu \mathrm{m}$ (Supplementary Table S3). The mean conidial length and width ranged from $11.5 \mu \mathrm{m}$ to $25.5 \mu \mathrm{m}$ and $4.0 \mu \mathrm{m}$ to $8.2 \mu \mathrm{m}$. The conidial morphology of Colletotrichum species on mango differed significantly between and within species. For examples, C. fructicola (GZ15-1) had profuse sporulation, C. gigasporum (HN42-2) produced the largest spores, and the shortest spores were generated by C. scovillei (YN51-1). 

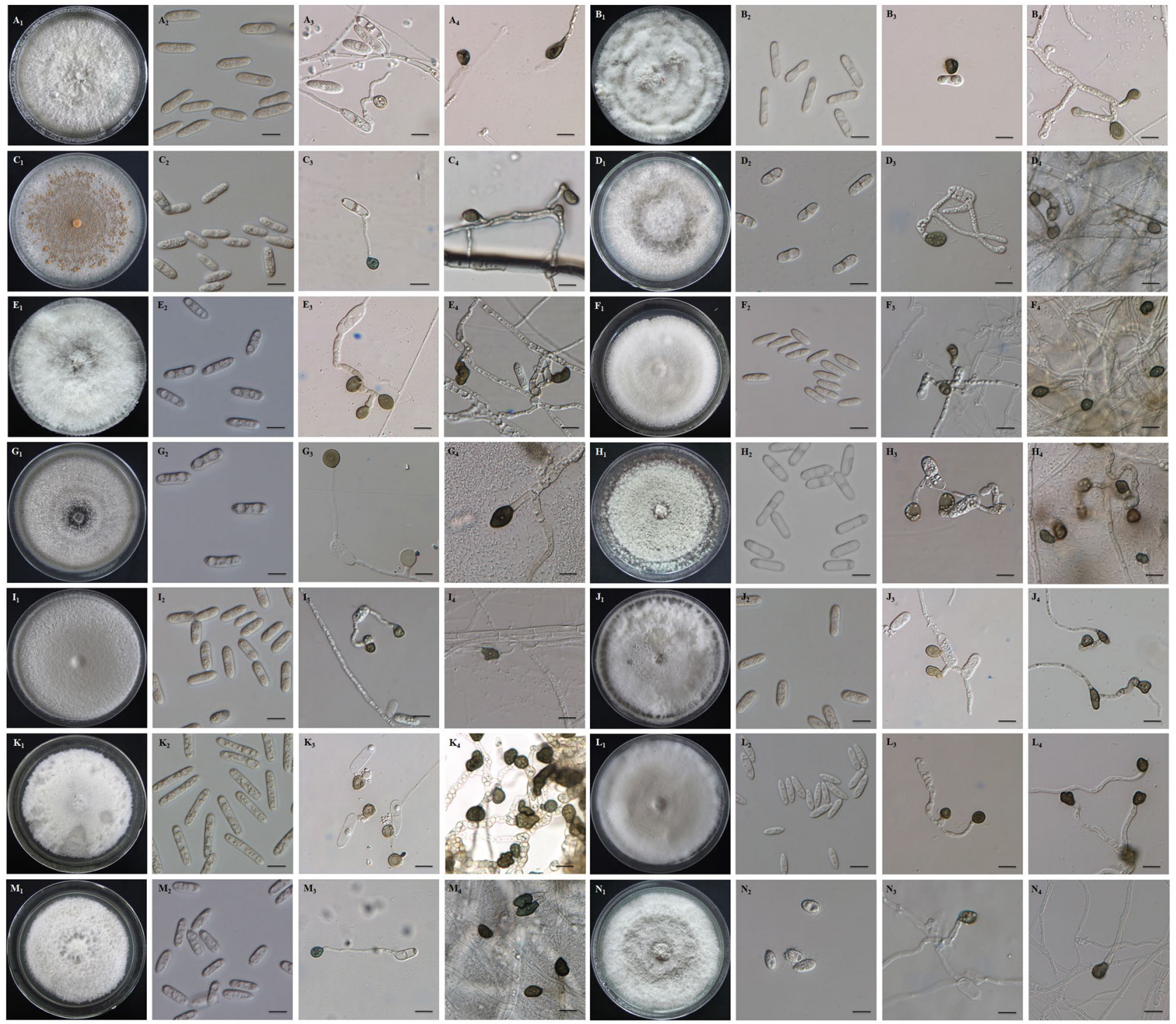

Figure 2. Morphological characteristics of colonies, conidia and appressoria of mango Colletotrichum isolates from China. Upper sides of (A1) C. asianum FJ18-3, (B1) C. asianum FJ31-6, (C1) C. siamense HN10, (D1) C. fruticola GZ15-1, (E1) C. karstii YN34-1, (F1) C. endophytica HN37-2, (G1) C. scovillei YN51-1, (H1) C. cliviicola YN8-2, (I1) C. gigasporum HN42-2, (J1) C. gloeosporioides GZ14-G-1, (K1) C. liaoningense YN33-1, (L1) C. musae GZ23-3, (M1) C. tropicale YN40-1-2, (N1) C. cordylinicola HN23-5 on PDA plates at 14 days after inoculation; Conidia of (A2) C. asianum FJ18-3, (B2) C. asianum FJ31-6, (C2) C. siamense HN10, (D2) C. fruticola GZ15-1, (E2) C. karstii YN34-1, (F2) C. endophytica HN37-2, (G2) C. scovillei YN51-1, (H2) C. cliviicola YN8-2, (I2) C. gigasporum HN42-2, (J2) C. gloeosporioides GZ14-G-1, (K2) C. liaoningense YN33-1, (L2) C. musae GZ23-3, (M2) C. tropicale YN40-1-2, (N2) C. cordylinicola HN23-5 on PDA plates after 14 days at $25^{\circ} \mathrm{C}$; Conidial appressoria of (A3) C. asianum FJ18-3, (B3) C. asianum FJ31-6, (C3) C. siamense HN10, (D3) C. fruticola GZ15-1, (E3) C. karstii YN34-1, (F3) C. endophytica HN37-2, (G3) C. scovillei YN51-1, (H3) C. cliviicola YN8-2, (I3) C. gigasporum HN42-2, (J3) C. gloeosporioides GZ14-G-1, (K3) C. liaoningense YN33-1, (L3) C. musae GZ23-3, (M3) C. tropicale YN40-1-2, (N3) C. cordylinicola HN23-5 on glass slides after 3 days at $25^{\circ} \mathrm{C}$; mycelial appressoria of (A4) C. asianum FJ18-3, (B4) C. asianum FJ31-6, (C4) C. siamense HN10, (D4) C. fruticola GZ15-1, (E4) C. karstii YN34-1, (F4) C. endophytica HN37-2, (G4) C. scovillei YN51-1, (H4) C. cliviicola YN8-2, (I4) C. gigasporum HN42-2, (J4) C. gloeosporioides GZ14-G-1, (K4) C. liaoningense YN33-1, (L4) C. musae GZ23-3, (M4) C. tropicale YN40-1-2, (N4) C. cordylinicola HN23-5 on cover slips after 7 days at $25^{\circ} \mathrm{C}$. Bars: $10 \mu \mathrm{m}$.

Conidial appressoria were grey cloud (\#B6B6B4) to black (\#000000), nearly elliptical or irregular. The length of appressoria for each species varied from $4.9 \mu \mathrm{m}$ to $14.9 \mu \mathrm{m}$ and the width of appressoria varied from $3.6 \mu \mathrm{m}$ and $10.6 \mu \mathrm{m}$ (Table 1). The mean length and width calculated for each isolate ranged from $6.4 \mu \mathrm{m}$ to $12.4 \mu \mathrm{m}$. Mycelial appressoria were pale brown to dark brown, rod-shaped, occasionally irregular. The length of appressoria for each species varied from $5.6 \mu \mathrm{m}$ to $20.7 \mu \mathrm{m}$ and the width of appressoria varied from $3.5 \mu \mathrm{m}$ to $15.4 \mu \mathrm{m}$. 


\begin{tabular}{|c|c|c|}
\hline Isolates & Size of conidial appressoria $(\text { Mean } \pm \mathrm{SD}, \mu \mathrm{m})^{\mathrm{a}}$ & Size of mycelial appressoria $($ Mean $\pm S D, \mu \mathrm{m})$ \\
\hline Colletotrichum assianum FJ18-3 & $8.55 \pm 0.51 \times 7.08 \pm 0.24$ & $8.85 \pm 0.25 \times 6.58 \pm 0.13$ \\
\hline C. siamense $\mathrm{HN} 10$ & $8.41 \pm 0.27 \times 6.22 \pm 0.44$ & $8.26 \pm 0.20 \times 6.32 \pm 0.20$ \\
\hline C. fructicola GZ15-1 & $8.39 \pm 0.49 \times 6.33 \pm 0.31$ & $7.78 \pm 0.13 \times 6.31 \pm 0.11$ \\
\hline C. karstiiYN 34-1 & $11.92 \pm 0.51 \times 7.35 \pm 0.22$ & $7.55 \pm 0.40 \times 5.66 \pm 0.11$ \\
\hline C. endophytica HN37-2 & $9.32 \pm 0.16 \times 7.10 \pm 0.18$ & $9.62 \pm 0.63 \times 5.72 \pm 0.26$ \\
\hline C. scovillei YN51-1 & $6.38 \pm 0.27 \times 4.91 \pm 0.19$ & $7.88 \pm 0.30 \times 5.45 \pm 0.14$ \\
\hline C. cliviicola YN8-2 & $8.94 \pm 0.29 \times 7.44 \pm 0.28$ & $11.15 \pm 0.94 \times 7.89 \pm 0.40$ \\
\hline C. gigasporum $\mathrm{HN} 42-2$ & $12.41 \pm 0.41 \times 9.36 \pm 0.34$ & $14.99 \pm 0.39 \times 12.21 \pm 0.34$ \\
\hline C. gloeosporioides GZ14-G-1 & $8.81 \pm 0.41 \times 5.26 \pm 0.19$ & $10.51 \pm 0.78 \times 5.94 \pm 0.24$ \\
\hline C. liaoningense YN33-1 & $7.03 \pm 0.15 \times 6.00 \pm 0.12$ & $10.47 \pm 0.34 \times 7.61 \pm 0.19$ \\
\hline C. musae GZ23-3 & $6.71 \pm 0.21 \times 6.09 \pm 0.21$ & $8.68 \pm 0.30 \times 6.63 \pm 0.19$ \\
\hline C. tropicale $\mathrm{YN} 40-1-2$ & $7.94 \pm 0.21 \times 6.98 \pm 0.22$ & $10.41 \pm 0.44 \times 6.62 \pm 0.16$ \\
\hline C. cordylinicola $\mathrm{HN} 23-5$ & $8.22 \pm 0.21 \times 5.15 \pm 0.18$ & $10.51 \pm 0.47 \times 6.44 \pm 0.27$ \\
\hline
\end{tabular}

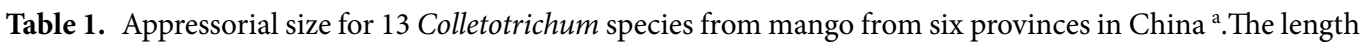
and width of 30 conidial appressoria and mycelial appressoria per isolate on glass and plastic slides were measured after 3 days and 7 days at $25^{\circ} \mathrm{C}$, respectively.

The mean value of length and width calculated for each isolate ranged from $7.6 \mu \mathrm{m}$ to $15.0 \mu \mathrm{m}$ and 5.5 to $12.2 \mu \mathrm{m}$, respectively.

The various Colletotrichum species reported here showed differences in both conidial and appressorial sizes and growth rates (Table 1 and Supplementary Table S3). Based on morphology observation and multi loci sequence analysis, 128 mango Colletotrichum isolates obtained in China can be classified into 13 species as follow: 38 isolates (29.7\%) C. asianum, 38 isolates (29.7\%) C. siamense, 32 isolates (25.0\%) C. fructicola, five isolates C. karstii (3.9\%), four isolates C. endophytica (3.1\%), three isolates C. scovillei (2.3\%), two isolates C. cliviicola (1.6\%) and one isolate for each of the following species: C. cordylinicola, C. gigasporum, C. gloeosporioides, C. liaoningense, C. musae and C. tropicale.

This is the first report for C. cliviicola, C. cordylinicola, C. endophytica, C. gigasporum, C. gloeosporioides, C. karstii, C. liaoningense, C. musae and C. tropicale associated with mango anthracnose in China. This is also the first report for C. cordylinicola, C. endophytica, C. gigasporum, C. liaoningense and C. musae associated with mango anthracnose worldwide.

Provincial geographic distribution of Colletotrichum species on mango in China. The diversity of species and geographical distribution for various Colletotrichum species associated with mango anthracnose across different provinces in China are shown in Fig. 3. Hainan and Yunnan had the most abundant and diverse Colletotrichum species on mango. Nine Colletotrichum species (C. asianum, C. cordylinicola, C. endophytica, C. fructicola, C. gigasporum, C. karstii, C. scovillei, C. siamense, and C. tropicale) were recovered from mango with anthracnose symptoms in Hainan province while eight species (C. asianum, C. cliviicola, C. endophytica, C. fructicola, C. karstii, C. liaoningense, C. scovillei and C. siamense) were obtained from mango in Yunnan. Four species (C. fructicola, C. gloeosporioides, C. karstii and C. musae) were associated with mango anthracnose in Guizhou province. Only two Colletotrichum species (C. asianum and C. siamense) were isolated in Sichuan and Guangdong provinces. C. asianum and C. fructicola were found in Fujian province. As a whole, C. asianum, C. fructicola, C. siamense were the dominant species widely spread across the major mango growing areas in southern China, accounting for $84.4 \%$ of all isolates. C. endophytica, C. gigasporum, C. gloeosporioides and C. liaoningense were only found on wild mango trees (Supplementary Table S1).

Pathogenicity and aggressiveness in tissues. All 128 tested isolates were pathogenic to wounded mango leaves and fruits. The aggressiveness of the tested isolates on mango fruits differed significantly, with lesion diameters ranging from $0.54 \mathrm{~cm}$ to $3.64 \mathrm{~cm}$ (Supplementary Table S3). Based on diameter sizes of the disease spots, aggressiveness was classified as strongly virulent $(\geqq 2 \mathrm{~cm})$, moderately virulent $(\geqq 1.0$ to $<2 \mathrm{~cm})$ or weakly virulent $(<1.0 \mathrm{~cm})$. Among the 128 isolates investigated, $66(52 \%)$ isolates were moderately virulent, $9(7 \%)$ isolates were strongly virulent, 53 (41\%) isolates were weakly virulent. The least virulent strain was C. siamense SC16, while the most virulent strain was C. siamense GD7-1 (Supplementary Table S3).

The aggressiveness of the tested isolates on mango leaves was also significantly different, and the diameters of disease lesions varied from $0.60 \mathrm{~cm}$ to $4.34 \mathrm{~cm}$ (Supplementary Table S3). The aggressiveness of the tested strains in mango differed considerably. According to the diameter size of the disease lesions, the aggressiveness scale can be classified as strongly virulent $(\geqq 2 \mathrm{~cm})$, moderately virulent $(\geqq 1.0$ to $<2 \mathrm{~cm})$ and weakly virulent $(<1.0 \mathrm{~cm})$. Using this scale, 84 isolates $(65 \%)$ were moderately virulent, 38 isolates $(30 \%)$ were strongly virulent, 6 isolates (5\%) were weakly virulent. The least virulent strain was C. siamense YN56-1-1, while the most virulent strain was C. siamense SC2-1 (Supplementary Table S3). 

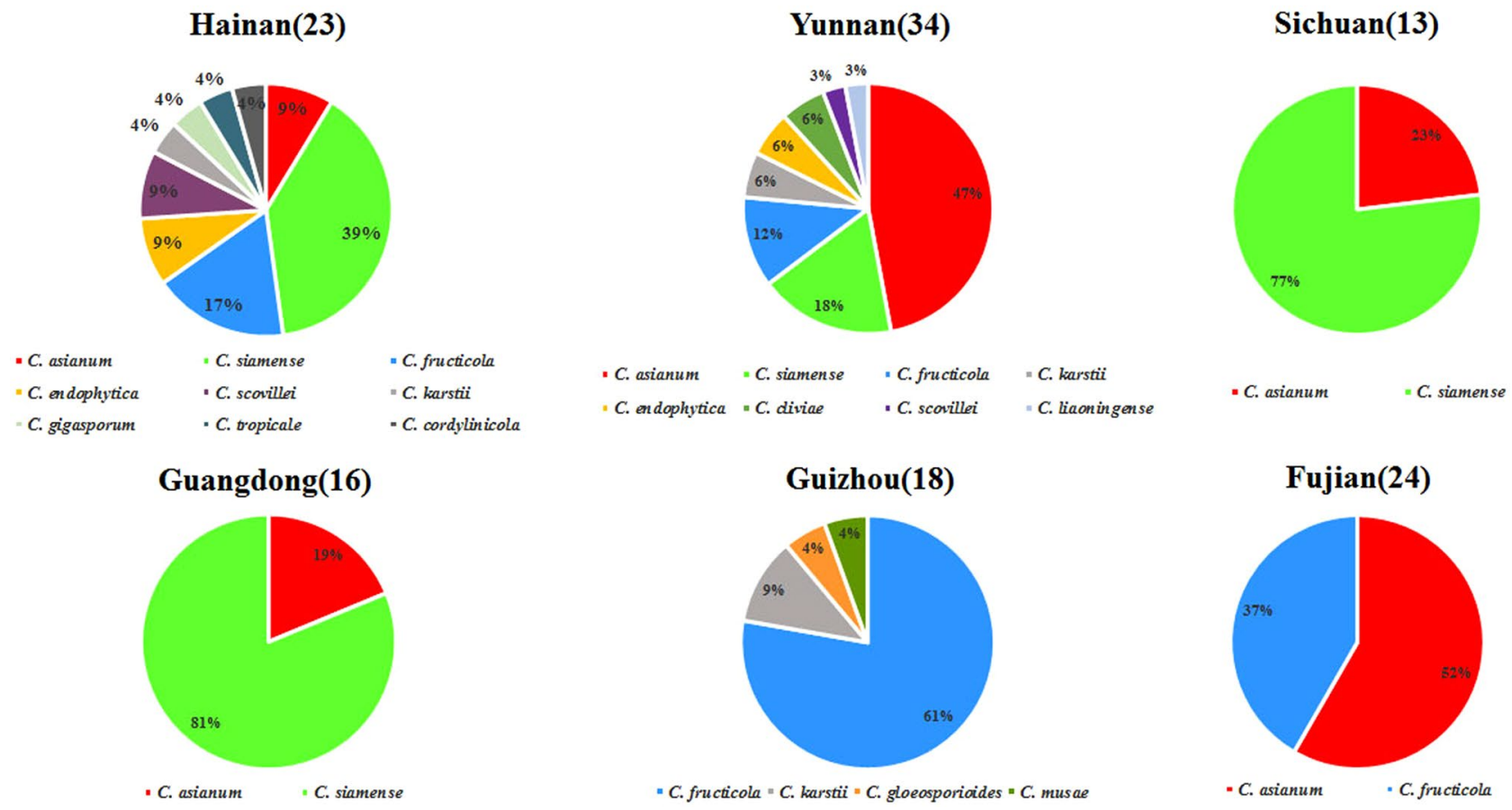

Figure 3. The percentage (\%) for dominant Colletotrichum species on mango isolated from Hainan, Yunnan, Sichuan, Guizhou, Guangdong and Fujian provinces in China with number of isolates from each province.

\section{Discussion}

In the past, common identification systems in Colletotrichum included traditional morphological taxonomy, molecular classification based on ITS sequences, presence of secondary metabolites and some other traits. These previously used identification systems alone or in combination were unable to fully resolve the species identity of individual Colletotrichum isolates ${ }^{6}$. Additional characteristics such as other aspects of morphology, and multi-gene phylogenetic analyses are needed to identify Colletotrichum species ${ }^{6}$.

Based on morphology and a concatenated five-gene phylogenetic analysis, 128 Colletotrichum isolates on mango from southern China were separated into 13 species: C. asianum, C. cliviicola, C. cordylinicola, C. endophytica, C. fructicola, C. gigasporum, C. gloeosporioides, C. karstii, C. liaoningense, C. musae, C. scovillei, C. siamense, and C. tropicale. Four species complexes (C. acutatum, C. boninense, C. gigasporum and C. gloeosporioides) were found to be associated with mango. The morphological descriptions of the isolates were in agreement with previous descriptions of Colletotrichum species, although cultural characteristics may be diverse due to varied cultural conditions, and conidial morphology alone could not distinguish all species within species complexes ${ }^{9}$.

Currently, thirteen Colletotrichum species have been reported worldwide on mango: C. asianum ${ }^{17}$, C. cliviicola $^{18}$, C. dianesei ${ }^{18}$, C. endomangiferae ${ }^{18}$, C. fructicola ${ }^{17}$, C. gloeosporioides ${ }^{19}$, C. $_{\text {grossum }}{ }^{20}$, C. kahawae $^{21}$, C. karstii $^{18}$, C. scovillei ${ }^{22}$, C. siamense ${ }^{23}$, C. theobromicola (syn. C. fragariae) ${ }^{19}$ and C. tropicale ${ }^{18}$. Mo et al. ${ }^{15}$, Liu et $a{ }^{24}$ and Qin et al. ${ }^{22,25}$ identified four Colletotrichum species (C. asianum, C. fructicola, C. scovillei, C. siamense) prevalent on mango in China. In this study, however, aside from C. asianum, C. fructicola, C. scovillei and C. siamense, there were 9 Colletotrichum species (C. cliviicola, C. cordylinicola, C. endophytica, C. gigasporum, C. gloeosporioides, C. karstii, C. liaoningense, C. musae, C. tropicale) which are the first reports on mango in China. This research also provides first reports for C. gigasporum, C. cordylinicola, C. musae, C. liaoningense and C. endophytica associated with mango world-wide.

The present study revealed high Colletotrichum species diversity associated with mango from the six major mango-grown areas in China. The Colletotrichum isolates examined here also showed high diversity, which may correlate with the diversity of environmental conditions such as temperature and rainfall ${ }^{26}$ and the sample collection time and isolate source. Colletotrichum asianum, C. fructicola and C. siamense were found to be the major species associated with mango anthracnose in China. This result agrees with what was reported by Vieira et al. ${ }^{18}$ (2014) who found that C. asianum was the most common endophytic species from mango trees. Colletotrichum asianum and C. siamense, were report as pathogens of a wide diversity of hosts such as Coffea arabica $a^{9}$, Carica papaya ${ }^{9}$ and $M$. indica ${ }^{15,19,27}$. Our study showed that a single host species may be attacked by several Colletotrichum species, and this was previously reported by Phoulivong et al. ${ }^{28}$. Colletotrichum fructicola was initially reported on coffee berries, and it also has a wide host range (Arachis sp., Citrus bergamia and M. indica) as well as wide geographical distribution ${ }^{29}$. Similarly, C. tropicale has been reported on Annona muricata (Annonaceae), Viola surinamensis (Myristicaceae) and M. indica in Brazil ${ }^{29}$.

Colletotrichum musae is a major causal agent of banana anthracnose and associated with fruit disease spots for Musa sp. in many countries ${ }^{29}$. Colletotrichum cliviicola was found to be associated with anthracnose of Clivia 
miniata, Cymbidium hookerianum in China ${ }^{12,30}$ and of Phaseolus sp. and Saccharum sp. in India ${ }^{12}$. It was also reported as an endophyte on $M$. indica in Brazil ${ }^{18}$.

Colletotrichum gloeosporioides was reported as a main pathogen from Citrus sp., Mangifera sp., and Vitis vinifera among many other host species, since it is considered a species complex ${ }^{9}$. It has a particularly wide host range based on publications because many Colletotrichum isolates were previously labelled C. gloeosporioides ${ }^{29}$. In the present study, C. asianum, C. cordylinicola, C. fructicola, C. gloeosporioides, C. kahawae, C. musae, C. siamense and C. tropicale, can all be placed into the C. gloeosporioides species complex ${ }^{9}$, and these results are consistent with previous reports that the C. gloeosporioides species complex is a preponderant Colletotrichum species associated with mango anthracnose $\mathrm{e}^{23,31}$.

Colletotrichm scovillei was reported as a pathogen on mango ${ }^{22}$ and Capsicum sp. (chilli "Django") ${ }^{32}$, and has been placed into the C. acutatum species complex ${ }^{10}$. Colleotrichum karstii was first reported from orchids and was also known from some other hosts such as Carica papaya ${ }^{11}$, Citrus sp. ${ }^{11}$, and M. indica ${ }^{11,17}$. Colletotrichum karstii has been found on mango and avocado fruit ${ }^{17,33}$, and it has been placed in the $C$. boninense species complex ${ }^{11}$.

Colletotrichum gigasporum belongs to the C. gigasporum species complex ${ }^{13}$. The species has large conidia and its wide range of hosts include Acacia auriculiformis and Coffea sp. (in Vietnam), and Diospyros kaki and Musa sp. (in Japan). Colletotrichum endophytica was found on Pennisetum purpureum in Thailand and tea plant in China ${ }^{34}$. Colletotrichum liaoningense was first reported as a pathogen of Capsicum annuum var. conoides in China ${ }^{35}$.

Pathogenicity test using thirteen species of Colletotrichum isolates showed that all species were pathogenic to mango leaves and fruits in the wound-inoculation experiment. The pathogenicity tests indicated that aggressiveness of Colletotrichum species on fruit was not completely consistent to that on leaves, but the aggressiveness of different isolates was found to be significantly different. The difference in aggressiveness of Colletotrichum within species was independent of a particular geographical origin for sample collection and varied, perhaps resulting from adaptations to the geographic and environmental origins of the isolates, although no specific correlations were found between aggressiveness and province of origin. However, there were more isolates from Yunnan province with weak aggressiveness compared to those from Hainan province. Symptoms may vary considerably with factors such as cultivar, fruit physiological state, inoculum factors such as concentration, and environmental conditions such as humidity and temperature ${ }^{33,36,37}$. Thus, the pathogenicity test results from this study may not reflect the full aggressiveness potential of the isolates examined. Additional research should be conducted to determine the aggressiveness for Colletotrichum species with conidial inoculation in planta rather than wounded detached leaves and fruits with mycelial plugs.

This study has enhanced our understanding on the diversity of Colletotrichum species associate with mango anthracnose in China. Further study is required to determine differences in biological and infection characteristics among various mango Colletotrichum species, as well as the molecular mechanisms responsible for such differences and their effects on mango anthracnose disease in the field. There have been few studies on the etiology and epidemiology of Colletotrichum species on mango. Therefore, future work on pathogenic mechanisms could be helpful for prevention and control of mango anthracnose disease.

\section{Materials and Methods}

Collection of sample and isolation of fungi. During 2016 and 2017, mango leaves, fruits and branches with symptoms or signs of anthracnose were gathered from six provinces (Fujian, Guangdong, Guizhou, Hainan, Sichuan and Yunnan) in China, covering an area $1950 \mathrm{~km}$ by $800 \mathrm{~km}$. Mango orchards were sampled with minimum $2 \mathrm{~km}$ distance between samples. Lesion margins on mango leaves, branches and fruits were cut into $3 \mathrm{~mm} \times 3 \mathrm{~mm}$ pieces, surface sterilized with ethanol (75\%) for $15 \mathrm{~s}$, sodium hypochlorite ( $1 \% \mathrm{vol} / \mathrm{vol})$ for $2 \mathrm{~min}$, followed by three rinses in autoclaved distilled water for $30 \mathrm{~s}$. Isolated fungus were cultured on potato dextrose agar (PDA) and kept at $25^{\circ} \mathrm{C}$. Over two weeks, the cultures were examined every day. Isolates were placed on PDA slants for longer term storage at $4^{\circ} \mathrm{C}$ in the dark.

Morphological characterization. Before morphological examination, isolates were cultured under continuous fluorescent light on PDA for week at $25^{\circ} \mathrm{C}$. Mycelial plugs $(5 \mathrm{~mm}$ diam) were cut from colony margins and placed 9-cm-diam Petri dishes with four plates per isolate. The colony characteristics (color of upper and lower surfaces) plus growth rate (diameters in two perpendicular directions) were recorded after 3, 5, 7 and 10 days. Hyphal growth rate (cm/day) was calculated based on colony diameter. Conidial production, shape and the size of conidia were examined up to three weeks on PDA incubated at $25^{\circ} \mathrm{C}$. Conidia ( 120 per isolate) from three-week-old PDA cultures were measured for length and width. Appressoria were produced from conidia placed on glass slides inside moist chambers. Appressoria were produced from hyphae using a slide culture technique described by Cai et al. ${ }^{6}$. PDA plugs $(1 \mathrm{~cm} \times 1 \mathrm{~cm})$ were placed in clean Petri dishes, and each agar plug was treated with conidia, and a cover slip was placed over each plug. After 7 days, the shape and size of the appressoria were recorded. For each isolate, at least 30 appressoria were examined, and photographs were taken under a Nikon Eclipse Ni-E microscope.

DNA extraction, polymerase chain reaction (PCR), and sequencing. Isolates were incubated at $25^{\circ} \mathrm{C}$ on PDA in Petri plates overlaid with cellophane. After 7 days of growth, mycelia were harvested by scraping the cellophane with autoclaved spatulas, and DNA was extracted using a CTAB method ${ }^{38}$. DNA was amplified with primers for partial actin (ACT), partial chitin synthase (CHS-1), partial glyceraldehyde-3-phosphate dehydrogenase (GAPDH), rDNA-ITS (ITS), and partial $\beta$-tubulin (TUB2) genomic regions (Supplementary Table S4). PCR amplification of ACT, CHS-1, GAPDH, TUB2 and ITS was amplified with the primer-pairs shown in Supplementary Table S4. PCR amplifications were done in 50 - $\mu$ l volumes containing $4 \mathrm{mM} \mathrm{MgCl}_{2}, 1 \times \mathrm{PCR}$ buffer, 0.5 units Taq DNA polymerase (Takara), $0.2 \mathrm{mM}$ concentrations of each dNTP, $0.5 \mu \mathrm{M}$ concentrations of 
each primer and $1 \mu \mathrm{l}$ of template DNA $(20 \mathrm{ng} / \mu \mathrm{l})$. The PCR programs for ACT, CHS-1, GAPDH, ITS and TUB2 were set following Mo et al. ${ }^{15}$ The PCR products were sequenced by Shanghai Sangon Company in China.

Phylogenetic analyses. Consensus sequences were obtained from forward and reverse primer sequencing of the same genomic region for each sequenced isolate using DNAMAN version 7.0. The consensus sequences were compared by BLASTn ${ }^{39}$ against the NCBI NR database. Among the top matches, select sequences of extype or ex-epitype isolates of Colletotrichum species were selected for the phylogenetic analyses (Supplementary Table S2). Alignments for each gene were carried out using MUSCLE v. 3.8.3 ${ }^{40}$. We tested whether the five gene data sets were combinable using the Partition Homogeneity Test as implemented in PAUP* 4.0b4 and described by Farris et al. ${ }^{41}$ as the incongruence length difference (ILD) test ${ }^{41}$. Sequences from the five genes were then manually concatenated and re-aligned. Phylogenetic analysis was conducted using MrBayes version 3.2.6 ${ }^{42}$. Models of nucleotide substitution for each gene determined by jModeltest v. 2.1.7 were included for each gene partition ${ }^{43}$. Following Drummond and Rambaut ${ }^{44}$ four runs with four chains for 5,000,000 generations were set up while the first $25 \%$ were discarded. The analyses were sampled every 1000 generations until the average standard deviation of split frequencies fell below 0.01. Maximum likelihood analyses were conducted with the RAxML GUI v. 1.3.1 ${ }^{45}$ using a GTRGAMMA substitution model, and bootstrap support was calculated based on 1,000 iterations.

Pathogenicity and aggressiveness on mango tissues. Pathogenicity tests were conducted following Mo et al. ${ }^{15}$. Select isolates were used for pathogenicity and aggressiveness tests on detached leaves and fruits of mango (cv. Tainong) under controlled conditions. Each isolate was incubated on PDA for $7-10$ days at $25^{\circ} \mathrm{C}$. Freshly harvested mango fruits and young leaves without visible disease from Tiandong (Guangxi province) were used for the tests. The tissues were surface sterilized in $75 \%$ ethanol for $30 \mathrm{~s}$ and $1 \% \mathrm{NaClO}$ for $1 \mathrm{~min}$, and then with three rinses in autoclaved distilled water.

After air drying, detached young healthy leaves $(12-15 \mathrm{~cm})$ were placed into plastic containers $(9 \mathrm{~cm} \times 17 \mathrm{~cm} \times 25 \mathrm{~cm})$ lined with paper towelling, and six stab wounds were made forming a 5-mm-diam circle. Mycelial plugs ( $6 \mathrm{~mm}$ diameter) from margins of PDA cultures were placed on each wound. The completely randomized trial with three replicates per isolate. For controls, sterile agar plugs were used. The containers were sealed and incubated in the dark at $25^{\circ} \mathrm{C}$. Aggressiveness was assessed one week after inoculation by measuring lesion diameter.

After washing and air drying, newly ripened mango fruits $(\sim 100 \mathrm{~g})$ were wounded by stabbing to a depth of $3 \mathrm{~mm}$ to form a 5-mm-diam circle. Mycelial plugs ( $6 \mathrm{~mm}$ diameter) from PDA cultures were placed on the wounds. Sterile agar plugs were used as control treatments. Inoculated fruits were placed in plastic containers on top of moist paper towelling and sealed. The containers were placed in a growth chamber and incubated at $25^{\circ} \mathrm{C}$ in the dark. Symptom development of the fruits was checked daily up to 10 days. Isolates were considered pathogenic when lesions expanded beyond the $5 \mathrm{~mm}$ circle of the initial wounding. Aggressiveness was evaluated at 10 days after inoculation (dpi) by measuring lesion diameter.

Statistical analyses. Statistical analyses were done using Data Processing System software (DPS 7.0) for Windows (Zhejiang University, Hangzhou, China) ${ }^{46}$, and data were subjected to analysis of variance (ANOVA) followed by Fisher's Least Significant Difference" (LSD) tests for means separation.

\section{Data availability}

All data can be available to Editorial Board Members, referees and readers.

Received: 4 January 2019; Accepted: 29 October 2019;

Published online: 11 December 2019

\section{References}

1. Kuhn, D. N. et al. Genetic map of mango: a tool for mango breeding. Front. Plant Sci. 8, 577 (2017).

2. Nan, N., Fu, Z. J. \& Xu, J. C. Mango industrialized development in China. Journal of Yunnan Agricultural University. 11, 80-84 (2017).

3. Hong, K. et al. Transcriptome characterization and expression profiles of the related defense genes in postharvest mango fruit against Colletotrichum gloeosporioides. Gene 576, 275-283 (2016)

4. Kamle, M. \& Kumar, P. Colletotrichum gloeosporioides: Pathogen of Anthracnose Disease in Mango (Mangifera indica L.). In Current Trends in Plant Disease Diagnostics and Management Practices. Springer, Cham. 207-219 (2016).

5. Hyde, K. D. et al. Colletotrichum: a catalogue of confusion. Fungal Divers. 39, 1-17 (2009).

6. Cai, L. et al. A polyphasic approach for studying. Colletotrichum. Fungal Divers. 39, 183-204 (2009).

7. Cannon, P. F., Damm, U., Johnston, P. R. \& Weir, B. S. Colletotrichum-current status and future directions. Stud. Mycol. 73, 181-213 (2012).

8. Freeman, S., Minz, D., Jurkevitch, E., Maymon, M. \& Shabi, E. Molecular analyses of Colletotrichum species from almond and other fruits. Phytopathology 90, 608-614 (2000).

9. Weir, B. S., Johnston, P. R. \& Damm, U. The Colletotrichum gloeosporioides species complex. Stud. Mycol. 73, 115-180 (2012).

10. Damm, U., Cannon, P. F., Woudenberg, J. H. C. \& Crous, P. W. The Colletotrichum acutatum species complex. Stud. Mycol. 73, 37-113(2012a).

11. Damm, U. et al. The Colletotrichum boninense species complex. Stud. Mycol. 73, 1-36 (2012b).

12. Damm, U., Sato, T., Alizadeh, A., Groenewald, J. Z. \& Crous, P. W. The Colletotrichum dracaenophilum, C. ámagnum and C. áorchidearum species complexes. Studies in mycology 92, 1-46 (2019).

13. Liu, F., Cai, L., Crous, P. W. \& Damm, U. The Colletotrichum gigasporum species complex. Persoonia 33, 83 (2014).

14. Sun., S. et al. Identification and biological characteristics of Colletotrichum asianum from post-harvest mango (Mangifera indica). Chinese Journal of Tropical Crops 37, 2392-2397 (2016).

15. Mo, J. et al. Identification and Characterization of Colletotrichum Species Associated with Mango Anthracnose in Guangxi, China. Plant Dis. 102, 1283-1289 (2018).

16. McDonald, B. A. \& Linde, C. The population genetics of plant pathogens and breeding strategies for durable resistance. Euphytica 124, 163-180 (2002). 
17. Lima, N. B. et al. Five Colletotrichum species are responsible for mango anthracnose in northeastern Brazil. Fungal Divers. 61, 75-88 (2013).

18. Vieira, W. A., Michereff, S. J., de Morais, M. A. Jr., Hyde, K. D. \& Câmara, M. P. Endophytic species of Colletotrichum associated with mango in northeastern Brazil. Fungal Divers. 67, 181-202 (2014).

19. Pardo De la Hoz, C. J. et al. Species from the Colletotrichum acutatum, Colletotrichum boninense and Colletotrichum gloeosporioides species complexes associated with tree tomato and mango crops in Colombia. Plant Pathol. 65, 227-237 (2016).

20. Leon, A. M. M., Hernández, W. S., Pérez, L. G., Crespo, K. \& Guarnaccia, V. First report of leaf anthracnose caused by Colletotrichum grossum on mango (Mangifera indica) in Cuba. J. Plant Pathol. 1, 1 (2018).

21. Ismail, A. M. et al. Characterisation of Colletotrichum species causing anthracnose disease of mango in Italy. J. Plant Pathol. 167-171 (2015).

22. Qin, L. P. et al. First Report of Anthracnose of Mangifera indica Caused by Colletotrichum scovillei in China. Plant Disease 103(5), $1043(2019)$.

23. Sharma, G., Kumar, N., Weir, B. S., Hyde, K. D. \& Shenoy, B. D. The ApMat marker can resolve Colletotrichum species: a case study with Mangifera indica. Fungal Divers. 61, 117-138 (2013).

24. Liu, L. P. et al. First report of post-harvest anthracnose on mango (Mangifera indica) caused by Colletotrichum siamense in China. Plant Disease 101(5), 833-833 (2017).

25. Qin, L. P., Huang, S. L., Lin, S. H. \& Lin, C. H. First report of anthracnose of Mangifera indica caused by Colletotrichum siamense in Sanya city in China. Plant Disease 101(6), 1038-1038 (2017).

26. Veloso, J. S., Câmara, M. P., Lima, W. G., Michereff, S. J. \& Doyle, V. P. Why species delimitation matters for fungal ecology: Colletotrichum diversity on wild and cultivated cashew in Brazil. Fungal Biol. 122, 677-691 (2018).

27. Lima, N. B., Lima, W. G., Tovar-Pedraza, J. M., Michereff, S. J. \& Camara, M. P. S. Comparative epidemiology of Colletotrichum species from mango in northeastern Brazil. Eur. J. Plant Pathol. 141, 679-688 (2015).

28. Phoulivong, S., McKenzie, E. H. C. \& Hyde, K. D. Cross infection of Colletotrichum species; a case study with tropical fruits. Current Research in Environmental \& Applied Mycology. 2, 99-111 (2012).

29. Jayawardena, R. S. et al. Notes on currently accepted species of Colletotrichum. Mycosphere 7, 1192-1260 (2016).

30. Yang, Y. L. et al. Colletotrichum anthracnose of Amaryllidaceae. Fungal Divers. 39(2), 123-146 (2009).

31. Arauz, L. F. Mango anthracnose: Economic impact and current options for integrated managaement. Plant disease 84(6), 600-611 (2000).

32. Cueva, F. D., Mendoza, J. S. \& Balendres, M. A. A new Colletotrichum species causing anthracnose of chilli in the Philippines and its pathogenicity to chilli cultivar Django. Crop Prot. 112, 264-268 (2018).

33. Giblin, F. R. et al. Colletotrichum species associated with pre-and post-harvest diseases of avocado and mango in eastern Australia. Australasian Plant Pathology 47(3), 269-276 (2018).

34. Wang, Y. C. et al. Diverse Colletotrichum species cause anthracnose of tea plants (Camellia sinensis (L.) O. Kuntze) in China. Sci. Rep. 6, 35-87 (2016).

35. Diao, Y. Z. et al. Colletotrichum species causing anthracnose disease of chili in China. Persoonia: Molecular Phylogeny and Evolution of Fungi 38, 20 (2017).

36. Freeman, S., Katan, T. \& Shabi, E. Characterization of Colletotrichum species responsible for anthracnose diseases of various fruits. Plant Dis. 82, 596-605 (1998).

37. Giblin, F. R., Coates, L. M. \& Irwin, J. A. G. Pathogenic diversity of avocado and mango isolates of Colletotrichum gloeosporioides causing anthracnose and pepper spot in Australia. Australasian Plant Pathology 39(1), 50-62 (2010).

38. Taylor, B. H., Manhart, J. R. \& Amasino, R. M. Isolation and characterization of plant DNAs. Methods in Plant Molecular Biology and Biotechnology. Glick, B. R. and Thompson, J. E. eds CRC Press, Boca Raton, FL (1993).

39. Altschul, S. F., Gish, W., Miller, W., Myers, E. W. \& Lipman, D. J. Basic local alignment search tool. J. Mol. Biol. 215, $403-410$ (1990).

40. Edgar, R. C. MUSCLE: multiple sequence alignment with high accuracy and high throughput. Nucleic acids research 32(5), 1792-1797 (2004).

41. Farris, J. S., Källersjö, M., Kluge, A. G. \& Bult, C. Testing significance of incongruence. Cladistics 10(3), 315-319 (1994).

42. Ronquist, F. et al. MrBayes 3.2: efficient Bayesian phylogenetic inference and model choice across a large model space. Systematic biol. 61, 539-542 (2012).

43. Darriba, D., Taboada, G. L., Doallo, R. \& Posada, D. jModelTest 2: more models, new heuristics and parallel computing. Nat. Methods 9, 772 (2012).

44. Drummond, A. J. \& Rambaut, A. BEAST: Bayesian evolutionary analysis by sampling trees. BMC Evol. Biol. 7, 214 (2007).

45. Silvestro, D. \& Michalak, I. raxmlGUI: a graphical front-end for RAxML. Organisms Diversity \&. Evolution 12(4), 335-337 (2012).

46. Tang, Q. Y. \& Zhang, C. X. Data Processing System (DPS) software with experimental design, statistical analysis and data mining developed for use in entomological research. Insect Sci. 20, 254-260 (2013).

\section{Acknowledgements}

The research was supported by National Natural Science Foundation of China (Grants No. 31600029, 31560526), Guangxi Natural Science Foundation (Grant No. 2016GXNSFCB380004), and Foundation for Development of Science and Technology Guangxi Academy of Agricultural Sciences (2018YM22).

\section{Author contributions}

Qili Li, Jianyou Mo and Zhihe Yu designed the experiments. Qili Li, Junyan Bu and Juan shu extracted DNA and done PCR. Lihua Tang, Suiping Huang, Jianyou Mo and Tangxun Guo performed morphological identification. Qili Li analyzed all the data. Shuming Luo, Ghulam Sarwar Solangi and Tom Hsiang revised the manuscript. This manuscript is not currently under consideration by any other journal, and the findings have not been published elsewhere. All authors have approved the submission of this manuscript.

\section{Competing interests}

The authors declare no competing interests.

\section{Additional information}

Supplementary information is available for this paper at https://doi.org/10.1038/s41598-019-54809-4.

Correspondence and requests for materials should be addressed to Z.Y. or J.M.

Reprints and permissions information is available at www.nature.com/reprints. 
Publisher's note Springer Nature remains neutral with regard to jurisdictional claims in published maps and institutional affiliations.

(c) (i) Open Access This article is licensed under a Creative Commons Attribution 4.0 International License, which permits use, sharing, adaptation, distribution and reproduction in any medium or format, as long as you give appropriate credit to the original author(s) and the source, provide a link to the Creative Commons license, and indicate if changes were made. The images or other third party material in this article are included in the article's Creative Commons license, unless indicated otherwise in a credit line to the material. If material is not included in the article's Creative Commons license and your intended use is not permitted by statutory regulation or exceeds the permitted use, you will need to obtain permission directly from the copyright holder. To view a copy of this license, visit http://creativecommons.org/licenses/by/4.0/.

(c) The Author(s) 2019 